\title{
PARECERISTAS AD HOC 2015
}

\section{AD HOC REVIEWERS 2015}

\section{Alessandra Rodrigues Prata Shimomura}

Universidade de São Paulo, São Paulo, Brasil

\section{Alex Kenya Abiko}

Universidade de São Paulo, São Paulo, Brasil

\section{Alfio Conti}

Universidade Federal de Ouro Preto, Ouro Preto, Brasil

Alice Theresinha Cybis Pereira

Universidade Federal de Santa Catarina, Florianópolis, Brasil

\section{Amelia de Farias Panet Barros}

Universidade Federal da Paraíba, João Pessoa, Brasil

Ana Claudia Veiga de Castro

Escola da Cidade, São Paulo, Brasil

Anja Pratschke

Universidade de São Paulo, São Paulo, Brasil

\section{Antonio Edesion Jungles}

Universidade Federal de Santa Catarina, Florianópolis, Brasil

\section{Antonio Manuel Nunes Castelnou Neto}

Universidade Federal do Paraná, Curitiba, Brasil

Carlos Eduardo Verzola Vaz

Universidade Federal de Pernambuco, Recife, Brasil

\section{Celina Lemos}

Universidade Federal de Minas Gerais, Belo Horizonte, Brasil

\section{Cibele Haddad Taralli}

Universidade de São Paulo, São Paulo, Brasil

Debora de Gois Santos

Universidade Federal de Sergipe, São Cristovão, Brasil

Douglas Queiroz Brandão

Universidade Federal do Mato Grosso, Cuiabá, Brasil
Eduardo Marques Arantes

Universidade Federal de Minas Gerais, Belo Horizonte, Brasil

\section{Eduardo Sampaio Nardelli}

Universidade Presbiteriana Mackenzie, São Paulo, Brasil

Elias Teodoro

Universidade Federal de Uberlândia, Uberlândia, Brasil

\section{Eloisa Dezen-Kempter}

Universidade Estadual de Campinas, Campinas, Brasil

\section{Ercília Hitomi Hirota}

Universidade Estadual de Londrina, Londrina, Brasil

Eugenio Fernandes Queiroga

Universidade de São Paulo, São Paulo, Brasil

\section{Evandro Ziggiatti Monteiro}

Universidade Estadual de Campinas, Campinas, Brasil

Fabiano Rogerio Corrêa

Universidade de São Paulo, São Paulo, Brasil

Flávia Brito do Nascimento

Universidade de São Paulo, São Paulo, Brasil

\section{Flávio Augusto Picchi}

Universidade Estadual de Campinas, Campinas, Brasil

\section{Gabriela Celani}

Universidade Estadual de Campinas, Campinas, Brasil

Gisela Leonelli

Universidade Estadual de Campinas, Campinas, Brasil

Giselle Arteiro Nielsen Azevedo

Universidade Federal do Rio de Janeiro, Rio de Janeiro, Brasil

\section{Glauco de Paula Cocozza}

Universidade Federal de Uberlândia, Uberlândia, Brasil

\section{Heliana Vargas}

Universidade de São Paulo, São Paulo, Brasil 
Jonathas Magalhães Pereira da Silva

Pontifícia Universidade Católica de Campinas,

Campinas, Brasil

\section{José de Paula Barros Neto}

Universidade Federal do Ceará, Fortaleza, Brasil

\section{José Merlin}

Pontifícia Universidade Católica de Campinas,

Campinas, Brasil

\section{José Paliari}

Universidade Federal de São Carslos, São Carlos, Brasil

\section{Lauro Luiz Francisco Filho}

Universidade Estadual de Campinas, Campinas, Brasil

\section{Leandro Medrano}

Universidade de São Paulo, São Paulo, Brasil

\section{Leonardo Marques Monteiro}

Universidade de São Paulo, São Paulo, Brasil

\section{Loyde Vieira de Abreu-Harbich}

Universidade Católica de Santos, Santos, Brasil

\section{Lucila Chebel Labaki}

Universidade Estadual de Campinas, Campinas, Brasil

\section{Marcia Regina de Freitas}

Universidade Estadual Paulista Júlio de Mesquita Filho, Guaratingetá, Brasil

\section{Márcio Minto Fabricio}

Universidade de São Paulo, São Carlos, Brasil

\section{Maria Beatriz Medeiros Kother}

Pontifícia Universidade Católica do Rio Grande do Sul, Porto Alegre, Brasil

\section{Maria Solange Gurgel de Castro Fontes}

Universidade Estadual Paulista Júlio de Mesquita Filho, Baurú, Brasil

\section{Mário Augusto Tavares Russo}

Instituto Politécnico de Viana do Castelo, Viana do

Castelo, Portugral

\section{Mirza Maria Baffi Pellicciotta}

Museu de Arte Sacra de São Paulo, São Paulo, Brasil

Oscar Luís Ferreira

Universidade de Brasília, Brasília, Brasil
Paulo Roberto Pereira Andery

Universidade Federal de Minas Gerais, Belo Horizonte, Brasil

Guilherme Carlos Lassance dos Santos Abreu

Universidade Federal do Rio de Janeiro, Rio de Janeiro, Brasil

\section{Renato Tibiriçá de Saboya}

Universidade Federal de Santa Catarina, Florianópolis, Brasil

\section{Regiane Trevisan Pupo}

Universidade Federal de Santa Catarina, Florianópolis, Brasil

\section{Regina Andrade Tirello}

Universidade Estadual de Campinas, Campinas, Brasil

\section{Renato Cymbalista}

Universidade de São Paulo, São Paulo, Brasil

\section{Renato Leão Rego}

Universidade Estadual de Maringá, Maringá, Brasil

\section{Sandro Canavezzi de Abreu}

Universidade Federal de Uberlândia, Uberlândia, Brasil

\section{Sergio Scheer}

Universidade Federal do Paraná, Curitiba, Brasil

\section{Sidney Piochi Bernardini}

Universidade Estadual de Campinas, Campinas, Brasil

\section{Stael de Alvarenga Pereira Costa}

Universidade Federal de Minas Gerais, Belo Horizonte, Brasil

\section{Stelamaris Rolla Bertoli}

Universidade Estadual de Campinas, Campinas, Brasil

\section{Tatiana Gondim do Amaral}

Universidade Federal de Goiás, Goiania, Brasil

\section{Tomas Antonio Moreira}

Universidade de São Paulo, São Carlos, Brasil

Vanessa Gomes da Silva

Universidade Estadual de Campinas, Campinas, Brasil

Walter Guedes da Silva

Universidade Estadual de Mato Grosso do Sul, Campo Grande, Brasil 